\title{
A Clinical and Etiological Study of Anemia in Patients with Primary Hypothyroidism
}

\author{
${ }^{1} \mathrm{~B}$ Mamatha Patil, ${ }^{2} \mathrm{~V}$ Shreyas Kumar, ${ }^{3}$ Kotha Anusha
}

\begin{abstract}
Introduction: Hypothyroidism has significant hematological manifestations. Overt and subclinical hypothyroidism both are associated with anemia. This study was done to evaluate the clinical and etiological profile of anemia in patients with primary hypothyroidism.
\end{abstract}

Materials and methods: Based on the symptoms and hormonal assay, 200 primary hypothyroidism patients were subjected to detailed examination. Patients were investigated before the thyroid hormone replacement therapy.

Results: Hypothyroidism is more prevalent in women than in men. Higher percentage of hypothyroid women (54\%) suffer from anemia than hypothyroid men (45\%). Normocytic normochromic anemia $(58.49 \%)$ was the most common type of anemia followed by microcytic hypochromic anemia $(20.75 \%)$, macrocytic hypochromic anemia (12.26\%), and dimorphic anemia (8.49\%). Among the symptoms and signs elicited, the most common was generalized weakness seen among $49.5 \%$ of the subjects with clinical hypothyroidism, followed by weight gain $(34.5 \%)$, bradycardia $(27.5 \%)$, peripheral edema $(23 \%)$, menstrual irregularities in females (12.5\%), and cold intolerance seen in $12 \%$ of the study population. Few subjects presented with more than one sign and symptom. Majority of the subjects were investigated only after they presented with one of the signs and symptoms, hence falling under the category of clinical hypothyroidism (78\%). Antithyroid peroxidase (TPO) antibodies were present in only a minor percentage $(15 \%)$ of the study population, majority of them being in the 4th decade of life and incidence decreasing as age progressed probably because the mean age group of the study population was in the fourth decade of life. Women (17.41\%) had a slightly higher incidence of autoimmune hypothyroidism compared with men (13.63\%).

Conclusion: Hypothyroidism is common in females, maximum in the third decade probably because the mean age of the study population was 32.53 years. Majority of the patients $(53 \%)$ were diagnosed with anemia. Of them, majority presented with symptoms of hypothyroidism and were found to have normocytic normochromic anemia followed by microcytic hypochromic anemia. A systematic study was done to know the relationship of hypothyroidism with anemia and a study of the symptomatology of hypothyroidism. The identification of patients with hypothyroidism is an important individual and public health issue. Hence, early detection and initiation of

\footnotetext{
${ }^{1}$ Professor, ${ }^{2}$ Senior Resident, ${ }^{3}$ Postgraduate Student

${ }^{1-3}$ Department of General Medicine, RajaRajeswari Medical College \& Hospital, Bengaluru, Karnataka, India

Corresponding Author: B Mamatha Patil, Professor Department of General Medicine, RajaRajeswari Medical College \& Hospital, Bengaluru, Karnataka, India, e-mail: Dr. mamatharamesh@yahoo.in
}

hormone replacement therapy can minimize the incidence of associated anemia.

Keywords: Anemia, Antithyroid peroxidase antibodies, Hypothyroidism.

How to cite this article: Patil BM, Kumar VS, Anusha K. A Clinical and Etiological Study of Anemia in Patients with Primary Hypothyroidism. J Med Sci 2018;4(2):48-51.

Source of support: Nil

Conflict of interest: None

\section{INTRODUCTION}

The most common functional disorder of the thyroid gland is hypothyroidism. It is a clinical state due to the decreased secretion of thyroid hormones or more rarely, from their impaired activity at tissue level. Anemia and hypothyroidism are both common diseases in the community. ${ }^{1}$ We need to evaluate the prevalence and types of anemia in primary hypothyroid patients because both have significant medical implications. Therefore, this study was done to evaluate the clinical and etiological profile of anemia in patients with primary hypothyroidism. ${ }^{2}$

Hypothyroidism results from insufficient production of thyroid hormones, which decreases metabolism and leads to decreased tissue need for oxygen as an adaptation to hypometabolic state, leading to inadequate erythropoietin stimulation, thus causing anemia. ${ }^{3}$ In addition, malabsorption or nutritional deficiencies of iron, vitamin B12, and folate in hypothyroid state can aggravate or contribute to existing anemia. ${ }^{2}$

It is well known that hypothyroidism can cause various types of anemia, including microcytic, macrocytic, and normocytic anemia. Often, the first sign of hypothyroidism in many patients is anemia. It is also known that thyroid hormones can directly or indirectly stimulate the growth of erythroid colonies through erythropoietin.

\section{MATERIALS AND METHODS}

\section{Source of Data}

The study was conducted on patients attending the medicine outpatient department and patients admitted at all male and female medical wards of RajaRajeshwari 
Medical College and Hospital, Bengaluru, with clinical suspicion of hypothyroidism who fulfilled all the inclusion criteria and gave consent for the study for a period of 1 year from May 2015 to May 2016.

\section{Methods of Collection of Data}

A prospective study was conducted. The study comprises of a total of 200 cases of newly detected cases of subclinical and overt hypothyroidism patients who fulfill all the inclusion criteria. As there are no data available in RajaRajeswari Medical College \& Hospital and medical records section particularly regarding our study, by taking a sample size of 200 cases into account of prevalence, the power of the study will not be compromised with the sample size. Information is collected through predesigned pro forma for each patient. Detailed clinical evaluation of all patients [detailed history, detailed clinical examination supported by relevant clinical investigations where all patients undergo free triiodothyronine (T3), free thyroxine (T4) and thyroid-stimulating hormone (TSH) levels by chemiluminescence method and anti-TPO antibodies by chemical bioassay method along with hemoglobin levels, and peripheral smear] and other investigations, whenever indicated, were done.

\section{Inclusion Criteria}

All male and female patients with newly diagnosed subclinical (elevated TSH level 4-10 mL U/L with normal free T4 and free T3 levels) and overt (TSH level >10 mL U/L and low levels of free T4 and/or free T3) hypothyroidism cases above the age of 18 years who gave consent were included in this study.

\section{Exclusion Criteria}

- Patients with anemia due to other causes including known chronic diseases, infection, malignancy, hemolytic anemia, hemoglobinopathies, and bleeding diathesis.

- Subjects with clinical suspicion of any other endocrine system diseases other than hypothyroidism.

- Patients who were under the treatment that might affect blood parameters, such as steroids or who had received treatment for anemia previously.

- Patients with chronic kidney disease of any etiology.

- Patients with acute or chronic blood loss from the gastrointestinal tract, respiratory, genitourinary systems, and menorrhagia due to uterine pathology.

- Patients with prior thyroid disorder and/or treatment history.

- Pregnant women with hypothyroidism.

\section{RESULTS}

There are a total of 200 patients (Table 1). Among the total subjects 106 patients are anemic (Table 2). Among 200 patients, 22 male patients and 178 female patients are anemic (Table 3). Among the total 106 anemic patients, 10 are males and 96 are females (Table 4). A total of 200 subjects were studied in the present study, among which 157 subjects had clinical/overt hypothyroidism, 43 had subclinical hypothyroidism (Table 5). Among the total number of patients, 3 males and 31 females had positive anti-TPO antibodies (Table 6). Graph 1 represents genderwise distribution of symptoms and signs; 5 males and 19 females had cold intolerance; 9 males and 90 females

Table 1: Distribution of cases according to age and sex in the study population $(n=200)$

\begin{tabular}{llll}
\hline Age & Male & Female & Total \\
\hline $18-30$ & 4 & 89 & 93 \\
$31-40$ & 12 & 58 & 70 \\
$41-50$ & 5 & 25 & 30 \\
$51-60$ & 1 & 5 & 6 \\
$>60$ & 0 & 1 & 1 \\
\hline Total & 22 & 178 & 200 \\
\hline
\end{tabular}

Table 2: Number of total subjects diagnosed with anemia

\begin{tabular}{lll}
\hline Status & Total & Percent \\
\hline Anemic & 106 & 53 \\
Normal & 94 & 47 \\
\hline Total & 200 & 100 \\
\hline
\end{tabular}

Table 3: Number of male and female subjects diagnosed with anemia

\begin{tabular}{lllll}
\hline & $\begin{array}{l}\text { Male } \\
\text { (hemoglobin }\end{array}$ & & $\begin{array}{l}\text { Female } \\
\text { (hemoglobin }\end{array}$ \\
Status & $<14 \%)$ & Percent & $<12 \%)$ & Percent \\
\hline Anemic & 10 & 45.45 & 96 & 53.93 \\
Normal & 12 & 54.54 & 82 & 46.06 \\
\hline Total & 22 & 100 & 178 & 100 \\
\hline
\end{tabular}

Table 4: Distribution of types of anemia among anemic males and females in the study population

\begin{tabular}{lllll}
\hline Types of anemia & Males & Percent & Females & Percent \\
\hline NNA & 6 & 60 & 56 & 58.33 \\
MHA & 2 & 20 & 20 & 20.83 \\
MaH & 1 & 10 & 12 & 12.5 \\
Dim & 1 & 10 & 8 & 8.33 \\
\hline Total & 10 & 100 & 96 & 100 \\
\hline
\end{tabular}

NNA: Normocytic, normochromic anemia; MHA: Microcytic hypochromic anemia; MaH: Macrocytic, hyhpochromic anemia;

Dim: Dimorphic anemia

Table 5: Distribution of clinical and subclinical hypothyroidism

\begin{tabular}{ll}
\hline Type of hypothyroidism & Total $(n=200)$ \\
\hline Clinical/overt hypothyroidism & 157 \\
Subclinical hypothyroidism & 43 \\
\hline Total & 200 \\
\hline
\end{tabular}


Table 6: Distribution of autoimmune hypothyroidism among males and females in the study population

\begin{tabular}{llllll}
\hline $\begin{array}{l}\text { Anti-TPO } \\
\text { antibodies } \\
\text { in males }\end{array}$ & $n$ & Percent & $\begin{array}{l}\text { Anti-TPO } \\
\text { antibodies } \\
\text { in females }\end{array}$ & $n$ & Percent \\
\hline Positive & 3 & 13.63636364 & Positive & 31 & 17.41573034 \\
Negative & 19 & 86.36363636 & Negative & 147 & 82.58426966 \\
\hline Total & 22 & 100 & Total & 178 & 100 \\
\hline
\end{tabular}

had weakness; 7 males and 62 females had weight gain; 25 females had menstrual irregularities; 2 males and 44 females had edema and 4 males and 51 females had brady cardia.

\section{DISCUSSION}

Hypothyroidism is a common disease with varying frequency between countries. As decreased thyroid hormone adversely affects erythropoiesis, anemia develops in hypothyroidism. In our study, the female:male ratio was 8:1, suggesting a higher incidence in women compared with men which is known as recorded in previous literatures. ${ }^{4}$ The mean age of the study population was 32.53 years with peak incidence in the third decade of life.

According to the data of World Health Organization, anemia prevalence is $24.8 \%$ throughout the world and it is seen more frequently in underdeveloped countries. ${ }^{5} \mathrm{In}$ our study, $53 \%$ of the total study population was diagnosed to be anemic, which clearly indicates the role of hypothyroidism in the etiology of anemia as the number here is significantly higher than in the general population. Among the females in the study population, 53.93\% were diagnosed to be anemic, whereas among males, the number was $45.45 \%$, which is significantly lower when compared with females. In the present study, $49.5 \%$ of the study population presented with the complaint of generalized weakness, whereas a study conducted by
Watanakunakorn et al showed $69.75 \%$ of the study population presenting with the same complaint. ${ }^{6}$ In our study, $50.56 \%$ of females presented with this complaint, whereas only $40.90 \%$ of the males had this complaint.

The second most common complaint in our study was weight gain seen in $34.5 \%$ of the study population. In a study conducted by Watanakunakorn et al, $47 \%$ presented with weight gain, whereas Hira et al, in a study conducted, showed that $60 \%$ of their study population presented with this complaint. ${ }^{3}$ In our study, a slightly higher percentage of females (34.83\%) than males (31.81\%) presented with this complaint, hence ruling out any gender-related variations in weight gain. In a study conducted by Wayne et al, it was found that in $8 \%$ of the subjects studied, bradycardia was incidentally found. ${ }^{7}$ In a study conducted by William et al, the number was $13.3 \%$. In another study conducted by Anil Kumar et al, it was 14.3\%. In our study, we found that $27.5 \%$ of our study subjects had bradycardia, making it the most common sign in our study and showing a significantly higher percentage than previously conducted studies; $18.18 \%$ of the males and $28.65 \%$ of the females in our study were found to have bradycardia. In our study, $23 \%$ of the study population presented with the complaint of peripheral edema, whereas in the study conducted by Hira et al, the number was significantly higher at $45 \%{ }^{8}$ In our study, $24 \%$ of the females and $9 \%$ of the males presented with peripheral edema.

Due to increased health awareness today, patients presenting early to the hospital could have brought down this number, also indicating that peripheral edema is a late sign of hypothyroidism, probably coming after generalized weakness and weight gain as deduced by the percentage of subjects presenting with the abovementioned symptoms; $12 \%$ of the study population presented with the complaint of cold intolerance, with $22.72 \%$ of the males and $10.67 \%$ of the females presenting with this complaint. In the study conducted by Hira et al, cold

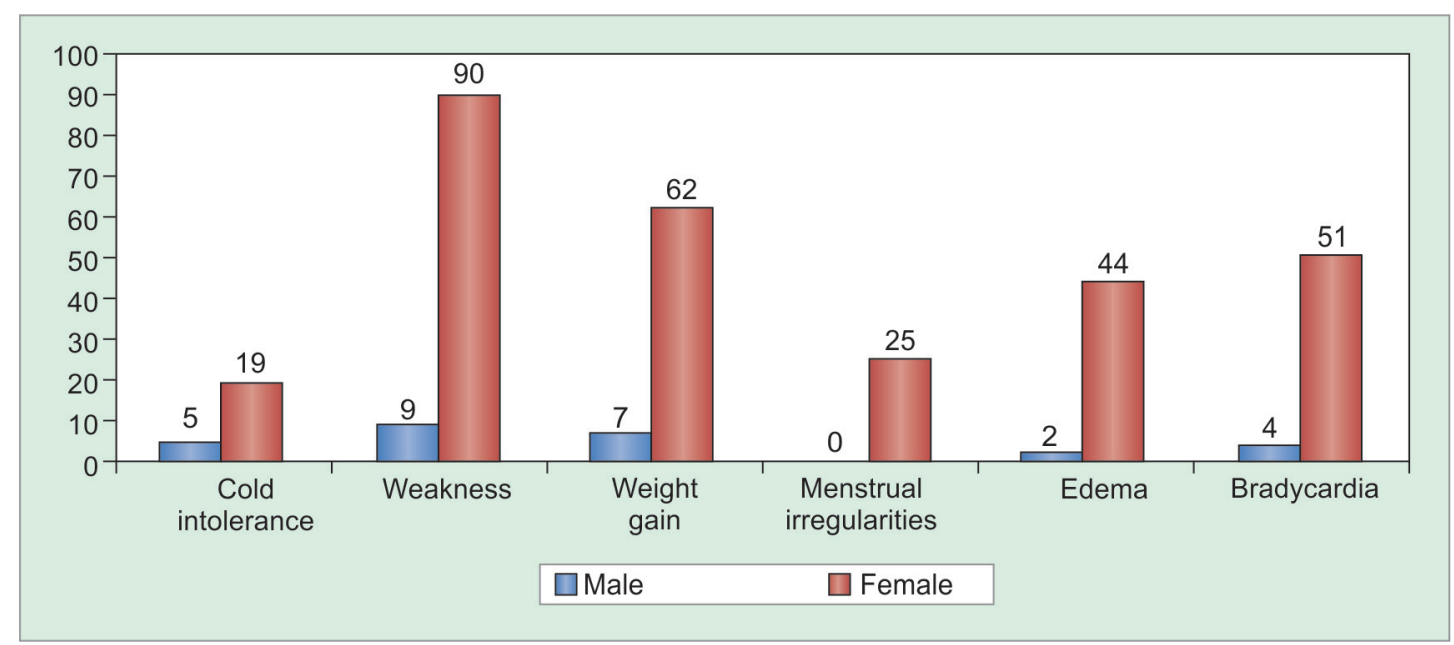

Graph 1: Gender-wise distribution of symptoms and signs 
intolerance was seen in $15 \%$ of the subjects. In the study conducted by Watanakunakorn et al, this number was significantly higher at 58\%. In our study, $10.67 \%$ females and $22.72 \%$ males presented with this complaint. Whether this can be attributed to higher amount of subcutaneous fat in women needs to be evaluated. Whether the general decrease in the percentage of people presenting with this complaint can be attributed to the overall decrease in average temperature (global warming) worldwide over the last 50 years needs further evaluation. About $12.5 \%$ of the female population studied presented with menstrual irregularities ranging from oligomenorrhea (5.6\%) to polymenorrhea (4.5\%) and menorrhagia (3.9\%).

Among the 200 subjects studied, 157 (78\%) presented with signs and symptoms of hypothyroidism, hence classified under clinical hypothyroidism and the rest 43 subjects (22\%) were incidentally diagnosed and classified as subclinical hypothyroidism. In our study, $17 \%$ of the study population was found to have anti-TPO antibodies positive, hence bringing them under the purview of autoimmune hypothyroidism. Peak incidence of autoimmune hypothyroidism was in the fourth decade of life and seen more in females where $17.41 \%$ were positive and among males, $13.63 \%$ were positive. In a study conducted by Chanchal Das et al, they found $58.3 \%$ to be having positive anti-TPO antibodies which is significantly higher than our present study. ${ }^{9}$ In our study, we found that $58.49 \%$ of the anemic subjects had normocytic normochromic anemia, $20.75 \%$ had microcytic hypochromic anemia, 12\% had macrocytic hypochromic anemia, and 9\% had dimorphic anemia. The percentage of prevalence among males and females was approximately the same in the study conducted by Chanchal Das et al; they found approximately the same percentage of subjects under the various types of anemia following the same order of hierarchy.

\section{CONCLUSION}

The hypothyroid patients present clinically with a myriad of symptoms and signs which are nonspecific. Hence, a high index of suspicion is the key for the early diagnosis of hypothyroidism. Hypothyroidism is more prevalent in women than in men. Higher percentage of hypothyroid women suffer from anemia than hypothyroid men.
Normocytic normochromic anemia is the most common type of anemia seen followed by microcytic hypochromic, macrocytic hypochromic, and dimorphic anemia, in that order in both men and women.

Among the signs and symptoms in both men and women, the most common was generalized weakness, followed by weight gain, bradycardia, peripheral edema, menstrual irregularities, and cold intolerance in that order. In males too, generalized weakness was followed by signs and symptoms in the same order excluding menstrual irregularities. Few subjects presented with more than one sign and symptom. Majority of the subjects were investigated only after they presented with one of the signs and symptoms, hence falling under the category of clinical hypothyroidism. Anti-TPO antibodies were present in only a minor percentage of the study population, majority of them being in the 4 th and 5 th decades of life and incidence decreasing as age progressed. Women had a slightly higher incidence of autoimmune hypothyroidism compared with men.

\section{REFERENCES}

1. Fein HG, Rivlin RS. Anemia in thyroid diseases. Med Clin North Am 1975;59:1133-1145.

2. DasC,SahanaPK,Sengupta N, GiriD, RoyM,MukhopadhyayP. Etiology of anemia in primary hypothyroid subjects in a tertiary care center in Eastern India. Indian J Endocr Metab 2012 Dec;16(Suppl 2):S361-S363.

3. Antonijevi N, Nesovi M, Trbojevi B, Milosevi R. Anemia in hypothyroidism. Med Pregl 1999;52(3-5):136-140 (in Croatian).

4. Worldwide Prevalence of Anaemia 1993-2005. WHO Global Database on Anaemia.

5. Lawrence E, Shapiro A, Surks MI. Hypothyroidism. In: Kenneth LB (ed.), Principles and practice of endocrinology and metabolism. 3rd ed. Philadelphia (PA): Lippincott Williams \& Wilkins; 2001. pp. 445-451.

6. Cinemre H, Bilir C, Gokosmanoglu F, Bahcebasi T. Hematologic effects of levothyroxine in iron-deficient subclinical hypothyroid patients: a randomized, double-blind, controlled study. J Clin Endocrinol Metab 2009;94(1):151-156.

7. Boelaert K, Franklyn JA. Thyroid hormone in health and disease. J Endocrinol 2005;187(1):1-15.

8. Reginad H, Maurice FS. Hypothyroidism: clinical features and complications. Clin Endocrinol Metabol 1979;8(1):29-38.

9. Roberts CG, Ladenson PW. Hypothyroidism. Lancet 2004 Mar 6;363(9411):793-803. 